\title{
Association of Socioeconomic Status With Breslow Thickness and Disease-Free and Overall Survival in Stage I-II Primary Cutaneous Melanoma
}

\author{
Mario Mandalà, MD; Gian Lorenzo Imberti, MD; Dario Piazzalunga, MD; Maurizio Belfiglio, MD; \\ Giuseppe Lucisano, MSc; Roberto Labianca, MD; Lorenzo Marchesi, MD; Barbara Merelli, MD; \\ Silvana Robone, H Econom; Paola Poletti, MD; Laura Milesi, MD; and Carlo Tondini, MD
}

\begin{abstract}
OBJECTIVE: To investigate the influence of socioeconomic status (SES) on Breslow thickness, disease-free survival, and overall survival in patients with stage I-II primary cutaneous melanoma (PCM).

PATIENTS AND METHODS: The study consists of all consecutive patients who were diagnosed as having PCM and were treated and followed up at our hospital between November 1, 1998, and July 31, 2009. Pathologic and sociodemographic characteristics of the patients were obtained. We categorized SES into 3 levels: low (manual employees and skilled/unskilled workers, including farmers, with primary education level), middle (nonmanual employees and clerks with middle education level), and high (professionals, executives, administrators, and entrepreneurs with tertiary education).
\end{abstract}

RESULTS: A total of 1443 consecutive patients were evaluated. In a multivariate logistic regression analysis, sex (female vs male: odds ratio [OR], 1.37; 95\% confidence interval [CI], 1.081.75), SES (high vs middle: OR, 1.27; $95 \% \mathrm{Cl}, 0.96-1.69$; high vs low: OR, 1.73; 95\% CI, 1.26-2.38), age ( $<60$ vs $\geq 60$ years: $0 R$, 1.35; $95 \% \mathrm{Cl}, 1.03-1.78$ ), and family context (single vs living with relatives: $0 \mathrm{R}, 1.37 ; 95 \% \mathrm{Cl}, 0.97-1.94)$ were the strongest correlates of Breslow thickness. Compared with high SES, the risk of melanoma-related death, adjusted for age and sex, was 7 times higher (hazard ratio, 7.44; 95\% Cl, 3.27-16.93) and almost 2 times higher (hazard ratio, 1.88; 95\% $\mathrm{Cl}, 1.04-3.39$ ) in patients with low SES living alone or living with relatives, respectively.

CONCLUSION: In patients with PCM, low SES is associated with thicker melanoma and a poorer clinical outcome.

Mayo Clin Proc. 2011;86(2):113-119

BT = Breslow thickness; $\mathbf{C l}=$ confidence interval; DFS = disease-free survival; $H R=$ hazard ratio; $O R=$ odds ratio; $O S=$ overall survival; PCM = primary cutaneous melanoma; $\mathbf{R E C P A M}=$ RECursive Partitioning and AMalgamation; SES = socioeconomic status

$\mathrm{T}$ The incidence of primary cutaneous melanoma (PCM) has been increasing steadily in the white population for several decades, doubling every 10 to 14 years; however, mortality figures show a much lower increase vs that recorded for incidence during the past 2 decades. ${ }^{1}$ The inverse relation between incidence and mortality may be explained by earlier detection of these tumors with thinner melanoma lesions at diagnosis because of improved public awareness regarding suspicious pigmented lesions. It is well demonstrated that Breslow thickness (BT) predicts both disease-free survival (DFS) and overall survival (OS). ${ }^{2}$ Socioeconomic status (SES) has been reported as a strong factor that may influence earlier detection of breast, head and neck, colon, and prostate cancer. ${ }^{3-7}$ Cancer pa- tients with a more disadvantaged SES have a higher risk of death compared with patients with a better SES. ${ }^{8}$

Much of the available evidence regarding melanoma is from retrospective studies, national registries, and data collected in academic centers. ${ }^{9-11}$ Several reports have used national cancer registries, which are generally important sources of data for describing disparities in cancer burden among different social groups of patients. ${ }^{12}$ However, these databases are usually limited to general demographic characteristics such as age, sex, race, and ethnicity; they lack important information regarding education, current employment or occupational status, and family context. ${ }^{10-13}$ In the absence of individual socioeconomic characteristics, several authors have used "area-based information" by linking patients through country, zip code, and census tract.

Birch-Johansen et $\mathrm{al}^{10}$ and Geller et $\mathrm{al}^{11}$ reported that melanoma patients with lower SES may be more likely to die of their disease. However, the reported information was geographic and rural area-based, and the authors were not able to collect information on thickness of the lesion at diagnosis. Hence, the influence of SES or education grade on BT and melanoma-related outcome has not been evaluated prospectively at a single, specific institution. At the Ospedali Riuniti in Bergamo, Italy, we have been collecting all socioeconomic, pathologic, and demographic information in a prospective database for all consecutive patients diagnosed as having PCM who were treated and followed up at our hospital by a multidisciplinary team.

In the current prospective analysis of a large patient population, we report the clinical relevance and influence of SES and family context on BT and clinical outcome of PCM.

From the Unit of Medical Oncology (M.M., R.L., B.M., P.P., L.M., C.T.), Unit of Dermatology (G.L.I., L.M.), and Unit of Surgery (D.P.), Riuniti Hospital, Bergamo, Italy; Department of Clinical Pharmacology and Epidemiology, Consorzio Mario Negri Sud, S. Maria Imbaro, Italy (M.B., G.L.); and Centre for Health Economics, University of York, UK (S.R.).

This work was supported by the Outcome Research Project at the Department of Hematology and Oncology, Ospedali Riuniti, Bergamo, Italy.

Individual reprints of this article are not available. Address correspondence to Mario Mandalà, MD, Unit of Medical Oncology, Ospedali Riuniti Bergamo, Largo Barozzi 1, 24126 Bergamo, Italy (mariomandala@tin.it).

(C) 2011 Mayo Foundation for Medical Education and Research 


\section{PATIENTS AND METHODS}

This study includes all consecutive patients who have been diagnosed as having PCM and were treated and followed up by a multidisciplinary team at the Ospedali Riuniti between November 1, 1998, and July 31, 2009. Data have been prospectively collected in a database (Outcome Research in Oncology Project study) with information on demographics, medical history, diagnosis, surgical procedures, pathologic features, systemic therapies, and follow-up.

Eligible patients were those seen and treated at Ospedali Riuniti for histologically confirmed PCM, clinically negative lymph nodes, and no evidence of distant disease. Sentinel lymph node biopsy was planned only for patients with lesions greater than $1.0 \mathrm{~mm}$ in thickness or for patients with lesions of $1.0 \mathrm{~mm}$ or smaller if adverse histopathologic features, such as ulceration or Clark level IV/V, were present. Demographics, including age at diagnosis, sex, working status and type of employment at the time of diagnosis, education grade, and family context, were prospectively collected for all patients.

All patients underwent wide local excision with free margins of at least $1 \mathrm{~cm}$ with a BT of $1 \mathrm{~mm}$ or less, of at least 1 to $2 \mathrm{~cm}$ with a BT of 1 to $2 \mathrm{~mm}$, and of at least $2 \mathrm{~cm}$ with a BT of $2 \mathrm{~mm}$ or more. Patients with positive results on sentinel lymph node biopsy were eventually offered radical lymphadenectomy and are not included in the current study. Systemic adjuvant therapy was considered for selected patients with nodal involvement.

Postoperative follow-up consisted of physical examinations, abdominal ultrasonography, chest radiography, and serum lactate dehydrogenase levels. Further investigations, including computed tomography, magnetic resonance imaging, and/or positron emission tomography, were selectively performed to investigate abnormal clinical findings that suggested the presence of metastatic melanoma. Routine surveillance was planned every 4 months for the first 2 years, every 6 months for years 3 to 5 , and annually thereafter. Postoperative follow-up was the same for patients with low, middle, and high SES.

For the purpose of the current study and according to other experiences, we grouped SES into 3 levels: low (manual employees and skilled/unskilled workers, including farmers, with primary education level [5 years]), middle (nonmanual employees and clerks with secondary education level [8-12 years]), and high (professionals, executives, administrators, and entrepreneurs with tertiary level of education). ${ }^{14}$

The local ethical committee of our hospital approved this study.

\section{Pathologic Features}

All the pathologic features were assessed by an expert dermatopathologist and by a clinical dermatologist as described previously ${ }^{15}$ Features routinely examined included BT (measured in millimeters), Clark level, presence or absence of ulceration, regression, and histologic subtype. Pathologists were blinded to patients' socioeconomic characteristics.

\section{Statistical Analyses}

Baseline patient characteristics and BT were compared using Pearson $\chi^{2}$. Univariate and multivariate proportional odds logistic regressions were performed to identify clinical and socioeconomic features predictive for higher BT $(0-1,1-3, \geq 3 \mathrm{~mm})$ at diagnosis. In the univariate analysis model the following variables were tested: sex (female vs male), age ( $<60$ vs $\geq 60$ years), SES (high vs middle and low), and marital status (single vs not single). The multivariate model was constructed with a stepwise regression method for the variables tested in the univariate analysis. The proportional odds assumption was assessed through the score test. Results were expressed as odds ratios (ORs) with 95\% confidence intervals (CIs). Both DFS and OS were calculated from the date of diagnosis to the date of first recurrence, death, or last follow-up.

Both OS and DFS were calculated by Kaplan-Meier analysis, and the differences tested with the log-rank test. We evaluated the effect of SES on survival by multivariate Cox proportional hazards analysis, adjusting for the same set of covariates used for the proportional odds logistic model predicting BT.

To evaluate the interaction effects among the different variables and identify distinct and homogeneous subgroups of patients with different risks of death or recurrence, the RECursive Partitioning and AMalgamation (RECPAM) method was used. ${ }^{16}$ This tree-growing technique, after Cox proportional hazards regression, allows assessment of interactions among covariates and identification of distinct and homogeneous subgroups of patients in terms of survival rates for OS and DFS. ${ }^{17}$ At each partitioning step, the RECPAM algorithm automatically chooses the covariate and its best binary split to maximize the difference in the outcome of interest. The algorithm stops when user-defined conditions (stopping rules) are met. The variables tested in the RECPAM analysis are the same as those tested in the proportional odds logistic model. Patients' age and sex are set as global predictors. Risks are reported as sex- and age-adjusted hazard ratios (HRs) with 95\% CIs. Breslow thickness (reported as both a continuous and a categorical variable) according to RECPAM classes was compared using Kruskal-Wallis one-way analysis of variance and Mantel-Haenszel $\chi^{2}$ test. To underline the predictive ability of the RECPAM tree, plots of the Kaplan-Meier estimates 
of survival distribution for OS and DFS according to the RECPAM classes were performed.

All analyses were performed using SAS Language (Release 9.1, SAS, Cary, NC). For the RECPAM analyses, we used an SAS macro routine written by 2 of the authors (G.L., M.B.). All statistical tests are 2-sided; $P<.05$ was considered significant.

\section{RESULTS}

A total of 1443 patients were enrolled in the study. The median age of the patients was 51 years (range 15-96 years), and 771 patients $(55.5 \%)$ were female. The mean \pm SD BT was $1.2 \pm 2.4 \mathrm{~mm}$. Ulceration was present in 251 patients (18.1\%), and 1123 patients (77.8\%) had stage I PCM.

At univariate analysis, sex $(P=.007)$, age $(P<.001)$, BT $(P=.002)$, Clark level $(P=.006)$, and presence of ulceration $(P<.001)$ were significantly correlated with SES (Table 1$)$. Sex, age, SES, and family context were associated with BT (Table 2).

In the multivariate logistic regression analysis, the proportional odds assumption was not rejected by the score test $(P=.07)$ at $5 \%$ significance level, and some variables were related to BT. In particular, sex (female is reference; male: OR, 1.37; $95 \% \mathrm{CI}, 1.08-1.75)$, age $(<60$ years is reference; $>60$ years: OR, 1.35 ; 95\% CI, 1.03-1.78), SES (high is reference; middle: OR, 1.27; 95\% CI, 0.96-1.69; low: OR, $1.73 ; 95 \% \mathrm{CI}, 1.26-2.38$ ), and family context (single status is reference; living with relatives: OR, 1.37; 95\% CI, 0.971.94) were the strongest correlates of BT increasing. These finding were confirmed in a multivariate stepwise analysis (Table 2).
Analyses relative to OS and DFS were conducted for 1355 patients with at least 1 follow-up visit and information about SES; median follow-up was 3.5 years.

The SES was the most significant predictor of DFS (Figure 1) and OS (Figure 2). Among patients of high, middle, and low SES, 10-year DFS was $91.8 \%, 90.8 \%$, and $81.7 \%$, respectively, and 10 -year OS was $97.3 \%, 98.3 \%$, and $91.6 \%$, respectively. Multivariate adjusted Cox proportional hazard model for OS and DFS in low SES confirmed these results (HR, 3.35; 95\% CI, 1.83-6.14; and HR, 1.88; 95\% CI, 1.32-2.69, respectively).

The RECPAM analysis identified 3 patient subgroups at different risk of both death and recurrence (Figures 3 and 4). According to the RECPAM algorithm, the most important variable in differentiating the risk of death was SES; in particular, patients with a middle/high SES were in a homogeneous class of risk with the lowest incidence of death and were considered the reference class (class 3 ). The risk progressively increased from class 3 to class 1 and was estimated with respect to the reference class.

Each of these classes showed a higher median value of BT. Notably, 11 patients (27.5\%) were diagnosed as having a BT greater than $3 \mathrm{~mm}$ in class 1 compared with 82 patients $(9.4 \%)$ in class 3 . The risk of melanoma-related death, adjusted for age and sex, was 7 times higher in patients with low SES and living alone (class 1: HR, 7.44; 95\% CI, 3.27-16.93) and almost 2 times higher in those with low SES but not living alone (class 2: HR, 1.88; 95\% CI, 1.04-3.39) (Figure 3). The RECPAM algorithm detected the same interaction for DFS. In fact, the risk of recurrence was almost 4 times higher (HR, 3.78; 95\% CI, 2.18-6.53) in class 1 (Figure 4).

TABLE 1. Characteristics of Patients With Primary Cutaneous Melanoma, Stratified by Socioeconomic Status (SES) ${ }^{\text {a }}$

\begin{tabular}{lccccc}
\hline & Low SES & Middle SES & High SES & Overall & $P$ value \\
\hline Age (y), mean \pm SD & $65.59 \pm 10.97$ & $48.89 \pm 14.68$ & $45.60 \pm 15.51$ & $51.65 \pm 16.42$ & $<.001$ \\
Sex & $213(60.5)$ & $241(55.7)$ & $317(51.5)$ & $771(55.1)$ & .007 \\
$\quad$ Female & $139(39.5)$ & $192(44.3)$ & $298(48.5)$ & $629(44.9)$ & \\
$\quad$ Male & $215(63.2)$ & $308(72.0)$ & $469(76.8)$ & $992(72.0)$ & .002 \\
Breslow thickness (mm) & $73(21.6)$ & $81(18.9)$ & $98(16.0)$ & $252(18.3)$ & \\
$0-1$ & $50(18.8)$ & $39(9.1)$ & $44(7.2)$ & $133(9.7)$ & \\
$1-3$ & & & & & \\
$>3$ & $50(15.1)$ & $62(14.7)$ & $101(16.9)$ & $213(15.8)$ & \\
Clark level & $107(32.2)$ & $147(34.8)$ & $248(41.4)$ & $502(37.1)$ & \\
1 & $65(19.6)$ & $108(25.6)$ & $132(22.0)$ & $305(22.5)$ & \\
2 & $98(29.5)$ & $96(22.8)$ & $111(18.5)$ & $305(22.5)$ & \\
3 & $12(3.6)$ & $9(2.1)$ & $7(1.2)$ & $28(2.1)$ & \\
4 & $244(74.2)$ & $347(82.4)$ & $508(86.8)$ & $1099(82.3)$ & $<.001$ \\
5 & $85(25.8)$ & $74(17.6)$ & $77(13.2)$ & $236(17.7)$ & \\
Ulceration & & &
\end{tabular}

${ }^{\mathrm{a}}$ Data are provided as number (percentage), unless otherwise indicated.

${ }^{\mathrm{b}} \chi^{2}$ Test. 
TABLE 2. Breslow Thickness Based on Sex, Age, SES, and Family Context

\begin{tabular}{lccc}
\hline Variable & $\begin{array}{c}\text { Univariate }^{\mathrm{a}} \\
\text { OR }(95 \% \mathrm{CI})\end{array}$ & $\begin{array}{c}\text { Multivariate }^{\mathrm{a}} \\
\text { OR }(95 \% \mathrm{CI})\end{array}$ & $\begin{array}{c}\text { Multivariate stepwise }^{\mathrm{a}} \\
\text { OR }(95 \% \mathrm{CI})\end{array}$ \\
\hline $\begin{array}{c}\text { Sex } \\
\text { Female }\end{array}$ & 1.00 & 1.00 & 1.00 \\
$\quad \begin{array}{c}\text { Male } \\
\text { Age (y) }\end{array}$ & $1.35(1.07-1.71)$ & $1.37(1.08-1.75)$ & $1.35(1.06-1.72)$ \\
$\quad<60$ & 1.00 & 1.00 & 1.00 \\
$\quad>60$ & $1.72(1.35-2.19)$ & $1.35(1.03-1.78)$ & $1.38(1.05-1.82)$ \\
SES & & & 1.00 \\
$\quad$ High & 1.00 & 1.00 & $1.26(0.95-1.68)$ \\
Middle & $1.26(0.66-1.67)$ & $1.27(0.96-1.69)$ & \\
$\quad$ Low & $1.94(1.46-2.59)$ & $1.73(1.26-2.38)$ & $1.72(1.25-2.36)$ \\
Lives alone & & & \\
$\quad$ No & 1.00 & 1.00 & \\
Yes & $1.40(0.69-1.98)$ & $1.37(0.97-1.94)$ & \\
\hline
\end{tabular}

${ }^{\text {a }}$ Odds logistic regression models. $\mathrm{CI}=$ confidence interval; OR = odds ratio; $\mathrm{SES}=$ socioeconomic status.

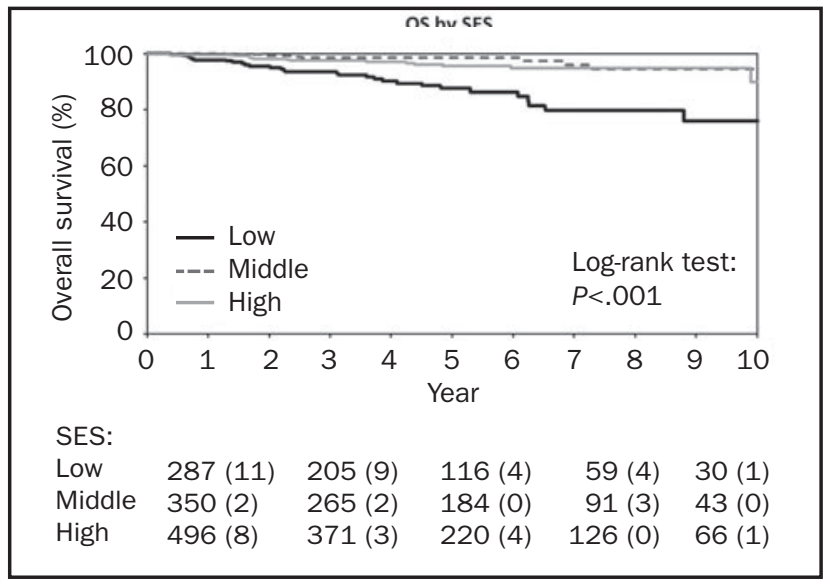

FIGURE 1. Socioeconomic status (SES) and overall survival in patients with stage I-II primary cutaneous melanoma. The numbers listed below the graph indicate the number (percentage) of events.

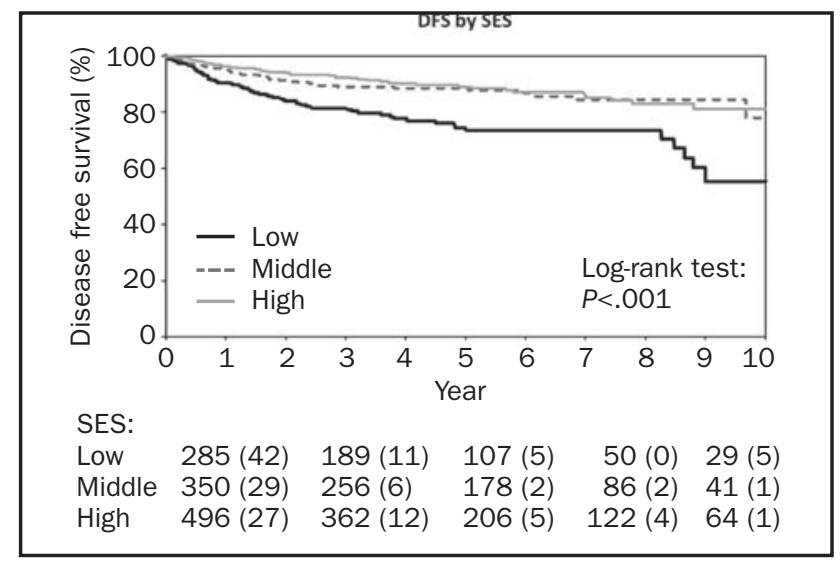

FIGURE 2. Socioeconomic status (SES) and disease-free survival in patients with stage I-II primary cutaneous melanoma. The numbers listed below the graph indicate the number (percentage) of events.

\section{DISCUSSION}

The results of the current study show a significant association between BT at diagnosis and age, sex, and indicators of SES, specifically education level and employment status. Furthermore, for patients with PCM, SES was related not only to BT at diagnosis but also to clinical outcome (DFS and OS). To our knowledge, this is the first article to report results by investigating a single institutional, prospectively collected database of patients with PCM. Of importance, all patients were diagnosed, treated, and evaluated at our hospital. All data were collected by a multidisciplinary clinical team, and pathologists involved in the diagnostic process were blinded to patient sociodemographic data. Furthermore, all occupational and education data were collected on an individual basis and were not from population registries.

In our patient population, results by multivariate logistic regression and multivariate stepwise analysis showed that men have a higher risk of being diagnosed with thicker melanomas. These findings are consistent with those from previous studies in which sex has been reported to have a significant role in the outcome of the disease. ${ }^{18,19}$ One possible hypothesis for these findings is that women devote more attention to their bodies and are more aware of health issues compared with men. However, biological differences between women and men cannot be ruled out. ${ }^{18}$ Women more frequently present with PCM in their extremities, whereas men have more trunk lesions, which are associated with poorer outcome.

Our data also showed that older age is significantly associated with thicker melanomas. Several explanations have been proposed for this finding. Elderly people usually give less importance to skin changes and perform self-examination less often compared with younger people..$^{20,21}$ Furthermore, age may affect host response to the disease; elderly patients are usually screened less often than younger patients and are more likely to be affected by nodular melanomas, which are known to have a high potential for metastases. ${ }^{18}$

In our study population, SES was associated with BT at diagnosis. The inclusion of these individual characteristics in the current analysis differentiates ours from previous studies. Our patients were stratified into 3 groups based on occupational position and education level as indicators of SES. This type of classification has been consistently reported in other studies. ${ }^{14}$ Moreover, these indicators have been used by the Italian National Statistical Institute (ISTAT, www.istat.it) to rank Italian population according to SES.

Our study results show that an increase in BT was associated with each of the aforementioned classes; in particular, $27.5 \%$ of patients in class 1 (low SES status and living alone) were diagnosed as having a BT greater than $3 \mathrm{~mm}$ compared with $9.41 \%$ for patients in class 3 (high 


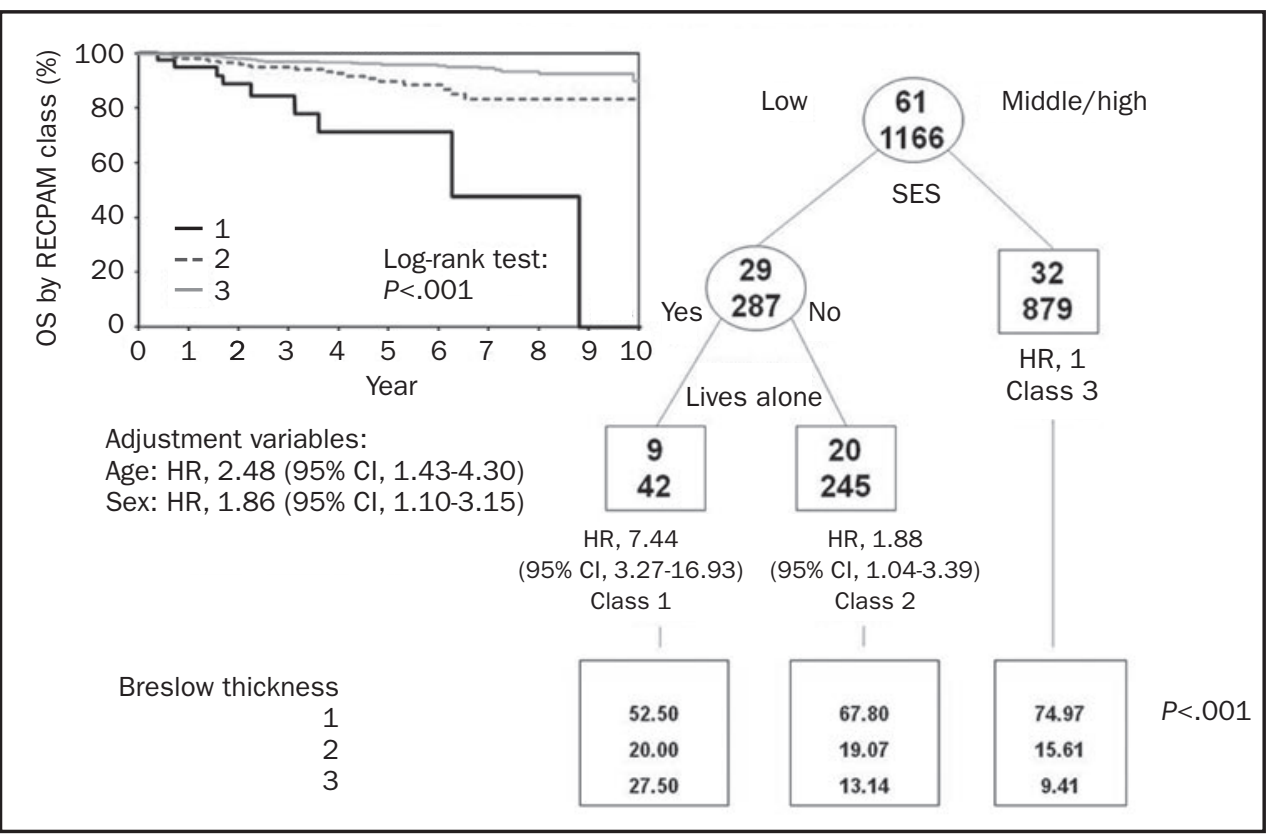

FIGURE 3. Identification of subgroups at different risks of overall survival (OS) based on results of RECursive Partitioning and AMalgamation (RECPAM) analysis. Tree-growing algorithm modeled hazard ratios (HRs) for both end points with a Cox regression model. Splitting variables (low SES vs middle/high SES and lives alone) are shown between branches, and the condition that sends patients to left or right branch is included. The class with the lowest incidence of events was set as reference category (HR, 1.00). Circles and squares indicate subgroups of patients. Numbers inside circles and squares represent patients with (top) and without (bottom) the event at issue. Under each RECPAM class, HRs represent the overall risk of event adjusted for age and sex compared with the reference class (class 3 ). $\mathrm{Cl}=$ confidence interval; SES = socioeconomic status.

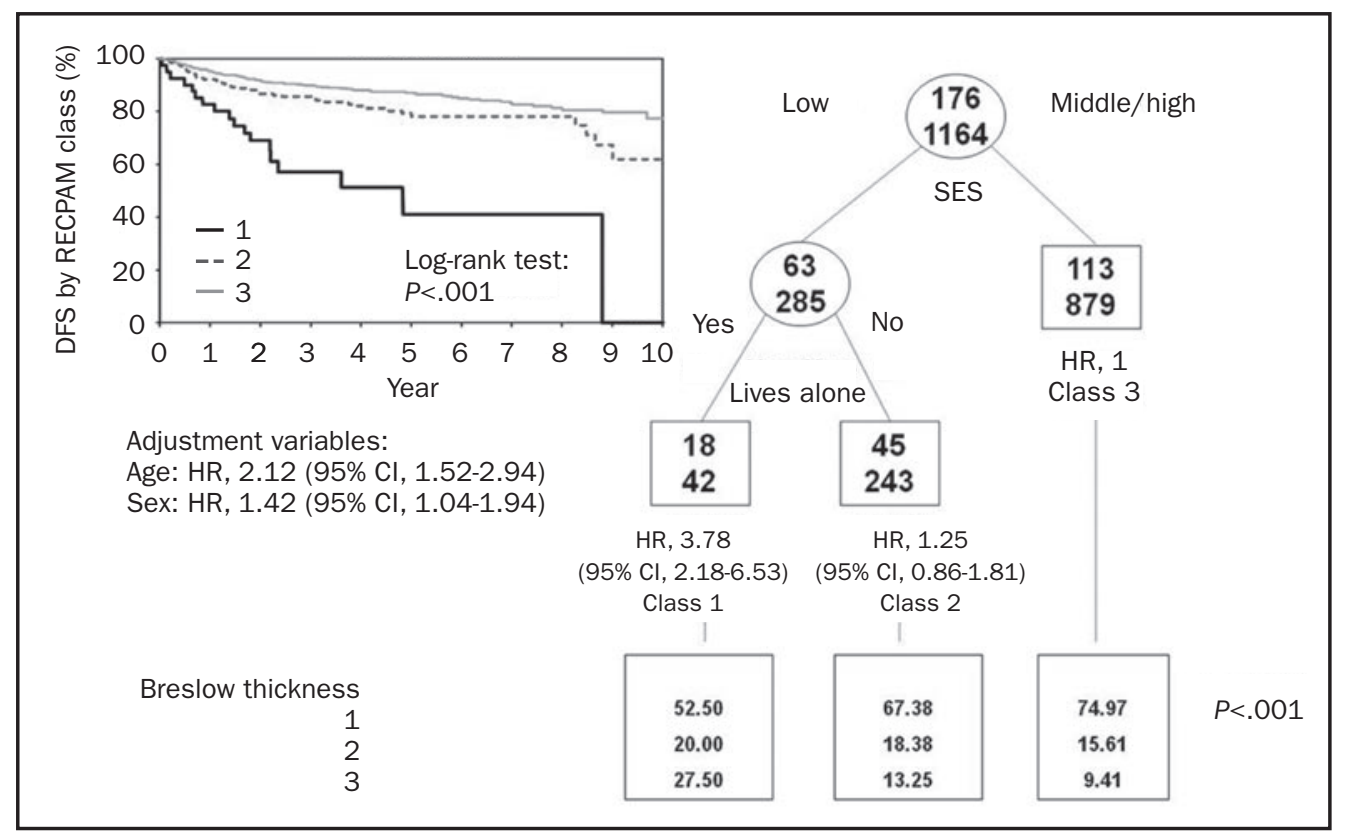

FIGURE 4. Identification of subgroups at different risks of disease-free survival (DFS) based on results of RECursive Partitioning and AMalgamation (RECPAM) analysis. Tree-growing algorithm modeled hazard ratios (HRs) for both end points with a Cox regression model. Splitting variables (low SES vs middle/high SES and lives alone) are shown between branches, and the condition that sends patients to left or right branch is included. The class with lowest incidence of events was set as reference category (HR, 1.00). Circles and squares indicate subgroups of patients. Numbers inside circles and squares represent patients with (top) and without (bottom) the event at issue. Under each RECPAM class, HRs represent the overall risk of event adjusted for age and sex compared with the reference class (class 3 ). $\mathrm{Cl}=$ confidence interval; SES = socioeconomic status. 
SES status). This strongly suggests that a late diagnosis is the key element for the worse prognosis in this group. Birch-Johansen et $\mathrm{al}^{10}$ recently reported that survival rates after a malignant melanoma are higher in those with a higher SES than for those with a lower SES. However, in that nationwide registry-based study, the authors could not adjust for BT.

To evaluate the interaction among the different variables and identify distinct and homogeneous subgroups of patients with different risks of death or recurrence, we used the RECPAM method. The risk of melanoma-related death in patients with low SES, adjusted for age and sex, was 7 times higher if living alone but was only 2 times higher if not living alone.

In our study, patients with middle and high SES seemed to overlap with regard to DFS and OS. There is no unique explanation for this finding. The fact that those with a low SES have a worse prognosis may be because the combination of low education level, poor family background, and lack of awareness leads to a diagnostic delay that adversely affects prognosis. In any case we cannot rule out that small differences do exist between patients with middle and high SES, which could be detected only by studying a larger cohort of patients. That is, to stress the importance of RECPAM tree-growing methods compared to main effects analysis, low SES was an homogeneous and distinct class of risk, whereas both middle and high SES were actually collapsed into one single risk class; this is a possible explanation of the reason why their survival curves overlapped.

Our study has some limitations. Because it is a single institutional experience in a single geographic area, our results may not necessarily apply to a different social context. Moreover, we could not analyze the influence of racial differences because all our patients were white. ${ }^{13,22}$ The lack of patients from other ethnicities is due to the fact that, until a few decades ago, Italy had minimal immigration. However, Italy has witnessed a substantial increase in immigration in the past few years, and future studies should take into account this change in the racial composition.

Previous studies have evaluated the role of health insurance coverage on the prognosis of melanoma, suggesting that differences in prognosis may be partially related to differences in health care access. ${ }^{23}$ Some health insurance companies may allow easier access to health care and may provide better facilities. Although Italy has a National Comprehensive Health Care system and health care is currently free of charge for all citizens, complementary private insurances are becoming more and more common among Italian citizens. Therefore, access to private insurance (much easier in advantaged socioeconomic groups) could be a factor that links socioeconomic inequalities to differences in health outcomes.
Our results have potential clinical implications. Our study suggests that there are groups of individuals in whom the diagnosis of melanoma will be delayed substantially. Because detection of PCM at an early stage is considered the most effective strategy to improve prognosis, identifying individuals with a higher chance of being diagnosed as having thicker and therefore prognostically less favorable PCM is important for targeting melanoma prevention campaigns. If further studies confirm our observations, future national strategies should take into account the importance of specific prevention campaigns involving people with low SES and education level to reduce cancer disparities and improve survival.

\section{CONCLUSION}

Evidence shows that cancer patients with a lower SES have a higher risk of death compared with patients with a higher SES. However, most of the studies reported only general demographic characteristics such as age, sex, race, and ethnicity. They lacked important information on education, current employment or occupational status, and family context.

We investigated the correlation between SES, education, family context, BT, DFS, and OS in patients with PCM. By using a prospective electronic database with individual data on pathologic and epidemiological features, we demonstrated that sex, age, SES, and family context are the strongest correlates of BT, the most important prognostic factor. Our findings show that, compared with melanoma patients with high SES, the risk of death due to melanoma was 7 times higher in patients with low SES and living alone and almost 2 times higher in patients with low SES but not living alone. Our data have clinical implications for the design of future melanoma prevention campaigns to reduce cancer disparities and improve the prognosis of more socioeconomically disadvantaged patients with melanoma.

This article is dedicated to our patients, who every day with their suffering teach us the difficult art of medicine.

\section{REFERENCES}

1. Jemal A, Siegel R, Ward E, et al. Cancer statistics, 2006. CA Cancer J Clin. 2006;56(2):106-130.

2. Balch CM, Soong SJ, Gershenwald JE, et al. Prognostic factors analysis of 17,600 melanoma patients: validation of the American Joint Committee on Cancer melanoma staging system. J Clin Oncol. 2001;19(16):3622-3634.

3. Tavani A, Fioretti F, Franceschi S, et al. Education, socioeconomic status and risk of cancer of the colon and rectum. Int J Epidemiol. 1999;28(3):380-385.

4. Li CI, Malone KE, Daling JR. Differences in breast cancer stage, treatment, and survival by race and ethnicity. Arch Intern Med. 2003;163(1):49-56.

5. Optenberg SA, Thompson IM, Friedrichs P, Wojcik B, Stein C, Kramer BS. Race, treatment, and long-term survival from prostate cancer in an equalaccess medical care delivery system. JAMA. 1995;274(20):1599-1605.

6. Shavers VL, Harlan LC, Winn D, Davis WW. Racial/ethnic patterns of care for cancers of the oral cavity, pharynx, larynx, sinuses, and salivary glands. Cancer Metastasis Rev. 2003;22(1):25-38.

7. Freedland SJ, Isaacs WB. Explaining racial differences in prostate cancer in the United States: sociology or biology? Prostate. 2005;62(3):243-252. 
8. Lantz PM, House JS, Lepkowski JM, et al. Socioeconomic factors, health behaviors, and mortality: results from a nationally representative prospective study of US adults. JAMA. 1998;279(21):1703-1708.

9. MacKie RM, Hole DJ. Incidence and thickness of primary tumours and survival of patients with cutaneous malignant melanoma in relation to socioeconomic status. BMJ. 1996;312(7039):1125-1128.

10. Birch-Johansen F, Hvilsom G, Kjoer T, Storm H. Social inequality and incidence of and survival from malignant melanoma in a populationbased study in Denmark, 1994-2003. Eur J Cancer. 2008;44(14):20432049.

11. Geller AC, Miller DR, Lew RA, et al. Cutaneous melanoma mortality among the socioeconomically disadvantaged in Massachusetts. Am J Public Health. 1996;86(4):538-543.

12. Bach PB, Guadagnoli E, Schrag D, Schussler N, Warren JL. Patient demographic and socioeconomic characteristics in the SEER-Medicare database applications and limitations. Med Care. 2002;40(8, suppl):IV-19IV-25.

13. Cormier JN, Xing Y, Ding M, et al. Ethnic differences among patients with cutaneous melanoma. Arch Intern Med. 2006;166(17):1907-1914.

14. Rapiti E, Fioretta G, Schaffar R, et al. Impact of socioeconomic status on prostate cancer diagnosis, treatment and prognosis. Cancer. 2009;115(23):5556-5565.

15. Mandala M, Imberti GL, Piazzalunga D, et al. Clinical and histopathological risk factors to predict sentinel lymph node positivity, disease-free and overall survival in clinical stages I-II AJCC skin melanoma: outcome analy- sis from a single-institution prospectively collected database. Eur J Cancer. 2009;45(14):2537-2545.

16. Ciampi A, Negassa A, Lou Z. Tree-structured prediction for censored survival data and the Cox model. J Clin Epidemiol. 1995;48(5):675-689.

17. Cox DR. Regression models and life-tables. J $R$ Stat Soc [Ser B]. 1972;B34:187-220.

18. Lasithiotakis K, Leiter U, Meier F, et al. Age and gender are significant independent predictors of survival in primary cutaneous melanoma. Cancer. 2008;112(8):1795-1804.

19. Scoggins CR, Ross MI, Reintgen DS, et al. Gender-related differences in outcome for melanoma patients. Ann Surg. 2006;243(5):693-698.

20. Austin PF, Cruse CW, Lyman G, Schroer K, Glass F, Reintgen DS. Age as a prognostic factor in the malignant melanoma population. Ann Surg Oncol. 1994;1(6):487-494.

21. Buettner PG, Leiter U, Eigentler TK, Garbe C. Development of prognostic factors and survival in cutaneous melanoma over 25 years: an analysis of the Central Malignant Melanoma Registry of the German Dermatological Society. Cancer. 2005;103(3):616-624.

22. Zell JA, Cinar P, Mobasher M, et al. Survival for patients with invasive cutaneous melanoma among ethnic groups: the effects of socioeconomic status and treatment. J Clin Oncol. 2008;26(1):66-75.

23. Halpern MT, Ward EM, Pavluck AL, et al. Association of insurance status and ethnicity with cancer stage at diagnosis for 12 cancer sites: a retrospective analysis. Lancet Oncol. 2008;9(3):222-231. 